\title{
Natural vibration analysis of pressurised and rotating toroidal shell segment by Rayleigh-Ritz method
}

\author{
Ivo Senjanović(1), Neven Alujević(1), Ivan Ćatipović(1), Damjan Čakmak ${ }^{(1)}$, Nikola \\ Vladimir $^{(1)}$, Dae-Seung Cho(2) \\ (1) University of Zagreb, Faculty of Mechanical Engineering and Naval Architecture, Zagreb, CROATIA \\ e-mail:ivo.senjanovic@fsb.hr \\ (2) Pusan National University, Department of Naval Architecture and Ocean Engineering, 63 beon-gil 2, Busandaehak-ro, \\ Geumjeong-gu, Busan, 46239, KOREA
}

\section{SUMMARY}

A semi-analytical procedure for natural vibration analysis of a simply supported toroidal shell segment is presented. The segment is closed in the circumferential direction and open in the meridional direction. The open edges are assumed to be simply supported. The segment therefore roughly resembles the geometry of a rotating tyre. The energy approach is used assuming shell displacements in the form of Fourier series in both circumferential and meridional directions. In the circumferential direction only one term of the Fourier series is necessary to describe exactly each vibration mode. In the meridional direction a relatively small number of Fourier series terms is necessary to achieve convergence. Tensional forces induced by the pressure and centrifugal loads are also determined by the energy approach. The application of the proposed procedure is illustrated by a numerical example. The increase in the internal pressure increases values of natural frequencies, whereas the shell rotation causes their bifurcation and the occurrence of travelling modes. In order to validate the method, the obtained results are compared with those determined by the finite strip method and by the finite element method.

KEY WORDS: $\quad$ toroidal shell; internal pressure; centrifugal load; natural vibration; RayleighRitz method.

\section{INTRODUCTION}

The theory of toroidal shell vibration is rather complicated due to the double curvature associated with the toroidal geometry $[1,2,3]$. Partial differential equations of motion can be derived directly, considering the equilibrium of sectional forces, inertial forces and external loads on an infinitesimal shell element. The equations can also be derived indirectly from the strain and kinetic energies of the complete shell, employing Hamilton's principle. However, 
due to the variable coefficients involved, it is not possible to obtain a closed form analytical solution. Therefore, numerical methods are ordinarily used, e.g. the finite element method (FEM) $[4,5,6]$.

Nowadays, vibration analysis of pre-stressed and rotating shells of revolution is becoming more and more relevant [7]. Such problems are found in engineering practice in rotor systems of gas turbine engines, high-speed centrifugal separators, rotating satellite structures, automotive tyres etc. $[8,9]$. Rotation makes dynamic behaviour of this type of structures significantly more complex due to bifurcation of natural frequencies and travelling natural modes [3]. Rotation speed is limited since it may imperil integrity of dynamic systems.

In case of complicated shell geometries, numerical methods like the FEM are, nowadays, normally used. However, analytical or semi-analytical methods offer a more transparent interpretation of results, save computational time, may increase accuracy and can be served as benchmarks for evaluation of numerical results.

Free vibration problem of rotating and pressurized closed toroidal shells has been solved by Rayleigh-Ritz method, by employing Fourier series [10]. In case of a closed toroidal shell, the variation of displacements in the circumferential direction can be described by Fourier series with only one term per vibration mode, whereas in the meridional direction it has been found that a relatively small number of terms is necessary to achieve good convergence [10]. Also, for an open toroidal shell, the finite strip method (FSM) has been applied, using a sophisticated toroidal finite strip which takes into account the effects of rotation and internal pressure [11, 12]. Additionally, special waveguide finite elements have been developed [13, 14]. As a result, a 3D problem can be reduced to a 2D problem, with significant computational time savings.

In this paper, a semi-analytical procedure for vibration analysis of a simply supported toroidal shell open in the meridional direction is given. The open edges are assumed to be simply supported. This geometry roughly approximates a rotating automotive tyre mounted onto a stiff wheel. The displacement field in both circumferential and meridional directions is assumed in the form of Fourier series. The Rayleigh-Ritz method is used. Tensional forces due to the internal pressure and the centrifugal load are determined in the same manner. The obtained results are compared with those determined by FSM and FEM.

\section{STRAIN AND KINETIC ENERGY OF ROTATING TOROIDAL SHELL}

The basic expressions for tensional and bending strain-displacement relationship of a toroidal shell are derived [10], using the general expressions of the shell theory. In this reference, vibration analysis of a toroidal shell with closed cross-section is performed by the RayleighRitz method and Fourier series. The strain energy is specified for large deformations and decomposed into a linear and a non-linear part. The non-linear part is also linearized afterwards. The kinetic energy is formulated taking into account a constant speed of rotation of the shell around its axis of symmetry. These are the starting equations for the subsequent vibration analysis of the toroidal shell segment in this paper.

An open toroidal shell with geometric parameters and displacement components is shown in Figure 1. The tensional displacement components in the meridional direction $u$, and the circumferential direction $v$, as well as the (bending) deflection $w$, are assumed in the following form: 


$$
\begin{aligned}
& u(\vartheta, \varphi, t)=U(\vartheta) \cos (n \varphi+\omega t) \\
& v(\vartheta, \varphi, t)=V(\vartheta) \sin (n \varphi+\omega t) \\
& w(\vartheta, \varphi, t)=W(\vartheta) \cos (n \varphi+\omega t),
\end{aligned}
$$

where functions $U(\vartheta), V(\vartheta)$ and $W(\vartheta)$ are the mode shape profiles in the meridional direction, and $\omega$ is the natural frequency. The argument $n \varphi+\omega t$ is used in order to describe travelling modes which normally appear due to shell rotation and $n$ is the circumferential mode number.
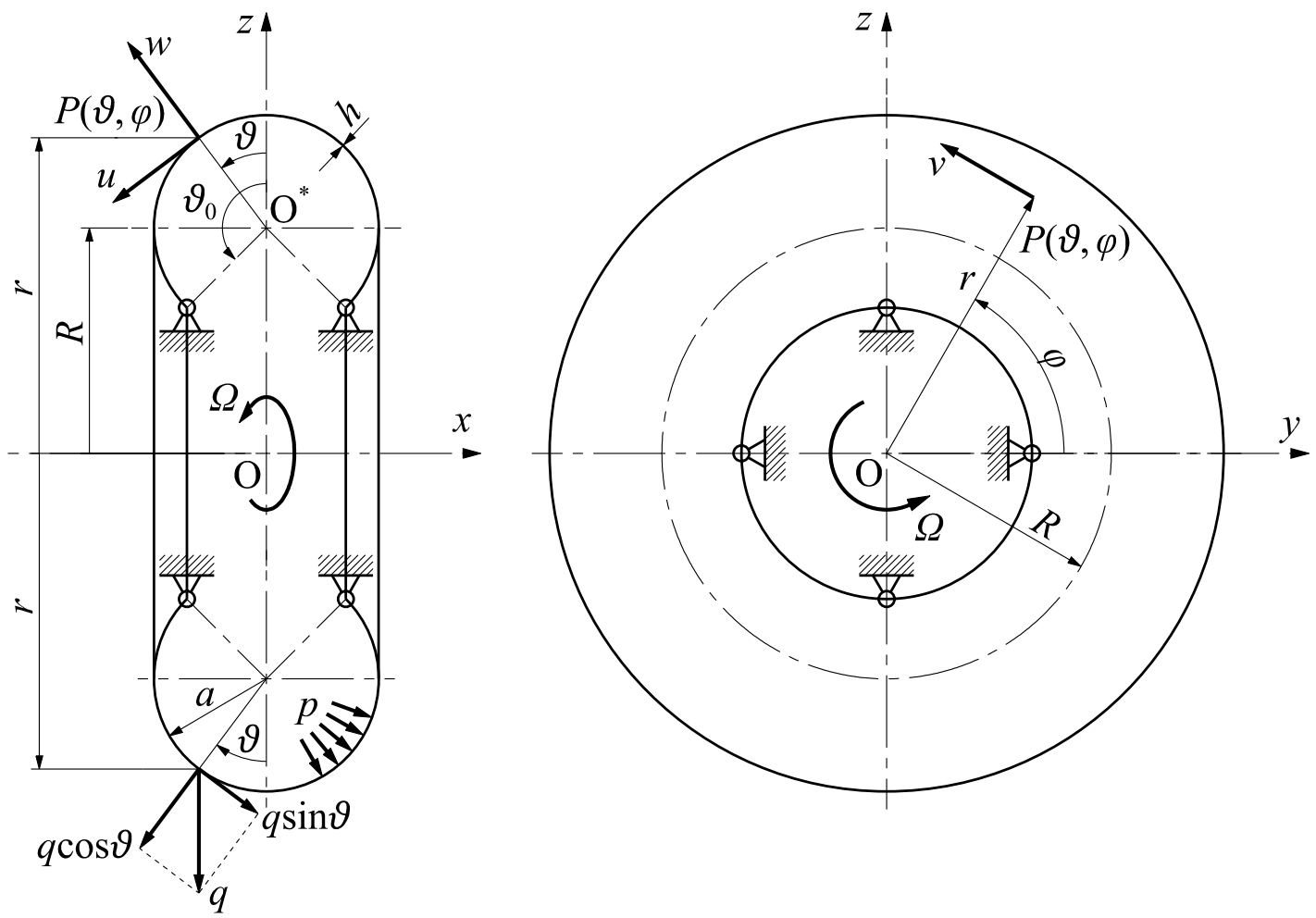

Fig. 1 Simply supported toroidal shell, main dimensions and displacements

Substituting expressions (1) into the corresponding formula for the strain energy from [10], and integrating in the circumferential direction, in the domain $0 \leq \varphi \leq 2 \pi$, one obtains:

$$
\begin{aligned}
E_{S}= & \int_{\vartheta}\left[\frac{1}{2} p_{1}\left(U^{\prime}\right)^{2}+\frac{1}{2} p_{2} U^{2}+p_{3} U^{\prime} U+\frac{1}{2} p_{4}\left(V^{\prime}\right)^{2}+\frac{1}{2} p_{5} V^{2}+p_{6} V^{\prime} V+\right. \\
& +p_{7} U^{\prime} V+p_{8} U V^{\prime}+p_{9} U V+ \\
& +\frac{1}{2} q_{1}\left(W^{\prime \prime}\right)^{2}+\frac{1}{2} q_{2}\left(W^{\prime}\right)^{2}+\frac{1}{2} q_{3} W^{2}+q_{4} W^{\prime \prime} W^{\prime}+q_{5} W^{\prime \prime} W+q_{6} W^{\prime} W+ \\
& +q_{7} W^{\prime \prime} U^{\prime}+q_{8}\left(W^{\prime \prime} U+W^{\prime} U^{\prime}\right)+q_{9} W^{\prime} U+q_{10} W U^{\prime}+q_{11} W U+ \\
& \left.+q_{12} W^{\prime \prime} V+q_{13} W^{\prime} V^{\prime}+q_{14} W^{\prime} V+q_{15} W V^{\prime}+q_{16} W V\right] d \vartheta,
\end{aligned}
$$

where $p_{i}(\vartheta), i=1,2 \ldots 9$ and $q_{j}(\vartheta), j=1,2 \ldots 16$ are variable coefficients specified in Appendix A. The primes designate spatial derivatives with respect to the meridional coordinate $\vartheta$.

In a similar way, using the general formulation for the strain energy due to pre-stressing [10], yields: 


$$
\begin{aligned}
E_{G}= & \int_{\vartheta}\left[\frac{1}{2} c_{1}\left(U^{\prime}\right)^{2}+\frac{1}{2} c_{2} U^{2}+\frac{1}{2} c_{3}\left(V^{\prime}\right)^{2}+\frac{1}{2} c_{4} V^{2}+c_{5} V^{\prime} V+\right. \\
& \left.+\frac{1}{2} c_{6}\left(W^{\prime}\right)^{2}+\frac{1}{2} c_{7} W^{2}+c_{8} U V+c_{9}\left(U^{\prime} W-U W^{\prime}\right)+c_{10} U W+c_{11} V W\right] d \vartheta,
\end{aligned}
$$

where $c_{i}(\vartheta), i=1,2 \ldots 11$ are variable coefficients specified in Appendix B.

The kinetic energy is presented in the form [10]:

$$
\begin{aligned}
E_{k}= & \frac{1}{2} \pi \rho h a \int_{\vartheta} r\left[\left(\omega^{2}+\Omega^{2} \sin ^{2} \vartheta\right) U^{2}+\left(\omega^{2}+\Omega^{2}\right) V^{2}+\left(\omega^{2}+\Omega^{2} \cos ^{2} \vartheta\right) W^{2}+\right. \\
& \left.+4 \omega \Omega(\cos \vartheta V W-\sin \vartheta U V)-2 \Omega^{2} \sin \vartheta \cos \vartheta U W\right] d \vartheta,
\end{aligned}
$$

where $\rho$ is the mass density, $h$ is the shell thickness, $a$ and $r=R+a \cos \vartheta$ are cross-sectional and circumferential radii, respectively, and $\Omega$ is the angular velocity of the shell rotation, Figure 1 .

All the three energy parts, Eqs. (2), (3) and (4) are time-invariant as a result of rotating modes having fixed circumferential profiles. For more details see, for instance $[7,10]$.

\section{SIMPLY SUPPORTED TOROIDAL SHELL SEGMENT}

\subsection{DISPLACEMENT FUNCTIONS}

For a simply supported segment of a toroidal shell, the meridional displacement functions can be assumed in the form of the following trigonometric series:

$$
\begin{aligned}
& U(\vartheta)=\sum_{m=1,3 \ldots}^{\infty} A_{m} \cos \xi_{m} \vartheta+\sum_{m=1,3 \ldots}^{\infty} B_{m} \sin \eta_{m} \vartheta \\
& V(\vartheta)=\sum_{m=1,3 \ldots}^{\infty} C_{m} \cos \xi_{m} \vartheta+\sum_{m=1,3 \ldots}^{\infty} D_{m} \sin \eta_{m} \vartheta \\
& W(\vartheta)=\sum_{m=1,3 \ldots}^{\infty} E_{m} \cos \xi_{m} \vartheta+\sum_{m=1,3 \ldots}^{\infty} F_{m} \sin \eta_{m} \vartheta,
\end{aligned}
$$

where

$$
\xi_{m}=m \frac{\pi}{2 \vartheta_{0}}, \quad \eta_{m}=(m+1) \frac{\pi}{2 \vartheta_{0}}
$$

are arguments of the trigonometric functions and $A_{m}, B_{m}, C_{m}, D_{m}, E_{m}$ and $F_{m}$ are the unknown Fourier coefficients.

Displacements (5) can be presented in the matrix notation as: 


$$
\begin{aligned}
& U(\vartheta)=\left\langle\left\langle f_{m}\right\rangle\left\langle g_{m}\right\rangle\right\rangle\left\{\begin{array}{l}
\left\{A_{m}\right\} \\
\left\{B_{m}\right\}
\end{array}\right\} \\
& V(\vartheta)=\left\langle\left\langle f_{m}\right\rangle\left\langle g_{m}\right\rangle\right\rangle\left\{\begin{array}{l}
\left\{C_{m}\right\} \\
\left\{D_{m}\right\}
\end{array}\right\} \\
& W(\vartheta)=\left\langle\left\langle f_{m}\right\rangle\left\langle g_{m}\right\rangle\right\rangle\left\{\begin{array}{l}
\left\{E_{m}\right\} \\
\left\{F_{m}\right\}
\end{array}\right\},
\end{aligned}
$$

where

$$
f_{m}=\cos \xi_{m} \vartheta, g_{m}=\sin \eta_{m} \vartheta
$$

\subsection{STIFFNESS MATRIX}

Substituting expressions (7) into (2) and differentiating the strain energy by the unknown coefficients, a system of three matrix equations is obtained:

$$
\begin{aligned}
& \left\{\begin{array}{l}
\frac{\partial E_{S}}{\left\{\partial A_{k}\right\}} \\
\left\{\partial B_{k}\right\}
\end{array}\right\}=\int_{-\vartheta_{0}}^{\vartheta_{0}}\left(p_{1}[k]_{1}+p_{2}[k]_{2}+p_{3}[k]_{3}\right) d \vartheta\left\{\begin{array}{l}
\left\{A_{m}\right\} \\
\left\{B_{m}\right\}
\end{array}\right\} \\
& +\int_{-\vartheta_{0}}^{\vartheta_{0}}\left(p_{7}[k]_{4}^{0}+p_{8}[k]_{4}^{*}+p_{9}[k]_{2}\right) d \vartheta\left\{\begin{array}{l}
\left\{C_{m}\right\} \\
\left\{D_{m}\right\}
\end{array}\right\} \\
& +\int_{-\vartheta_{0}}^{\vartheta_{0}}\left(q_{7}[k]_{5}^{0}+q_{8}\left([k]_{1}+[k]_{6}^{0}\right)+q_{9}[k]_{4}^{*}+q_{10}[k]_{4}^{0}+q_{11}[k]_{2}\right) d \vartheta\left\{\begin{array}{l}
\left\{E_{m}\right\} \\
\left\{F_{m}\right\}
\end{array}\right\} \\
& \left\{\begin{array}{l}
\frac{\partial E_{S}}{\left\{\partial C_{k}\right\}} \\
\left\{\partial D_{k}\right\}
\end{array}\right\}=\int_{-\vartheta_{0}}^{\vartheta_{0}}\left(p_{7}[k]_{4}^{*}+p_{8}[k]_{4}^{0}+p_{9}[k]_{2}\right) d \vartheta\left\{\begin{array}{l}
\left\{A_{m}\right\} \\
\left\{B_{m}\right\}
\end{array}\right\} \\
& +\int_{-\vartheta_{0}}^{\vartheta_{0}}\left(p_{4}[k]_{1}+p_{5}[k]_{2}+p_{6}[k]_{3}\right) d \vartheta\left\{\begin{array}{l}
\left\{C_{m}\right\} \\
\left\{D_{m}\right\}
\end{array}\right\} \\
& +\int_{-\vartheta_{0}}^{\vartheta_{0}}\left(q_{12}[k]_{6}^{0}+q_{13}[k]_{1}+q_{14}[k]_{4}^{*}+q_{15}[k]_{4}^{0}+q_{16}[k]_{2}\right) d \vartheta\left\{\begin{array}{l}
\left\{E_{m}\right\} \\
\left\{F_{m}\right\}
\end{array}\right\} \\
& \left\{\begin{array}{l}
\frac{\partial E_{S}}{\left\{\partial E_{k}\right\}} \\
\left\{\partial F_{k}\right\}
\end{array}\right\}=\int_{-\vartheta_{0}}^{\vartheta_{0}}\left(q_{7}[k]_{5}^{*}+q_{8}\left([k]_{1}+[k]_{6}^{*}\right)+q_{9}[k]_{4}^{0}+q_{10}[k]_{4}^{*}+q_{11}[k]_{2}\right) d \vartheta\left\{\begin{array}{l}
\left\{A_{m}\right\} \\
\left\{B_{m}\right\}
\end{array}\right\} \\
& +\int_{-\vartheta_{0}}^{\vartheta_{0}}\left(q_{12}[k]_{6}^{*}+q_{13}[k]_{1}+q_{14}[k]_{4}^{0}+q_{15}[k]_{4}^{*}+q_{16}[k]_{2}\right) d \vartheta\left\{\begin{array}{l}
\left\{C_{m}\right\} \\
\left\{D_{m}\right\}
\end{array}\right\} \\
& \left.+\int_{-\vartheta_{0}}^{\vartheta_{0}}\left(q_{1}[k]_{7}+q_{2}[k]_{1}+q_{3}[k]_{2}+q_{4}[k]_{8}+q_{5}[k]_{9}+q_{6}[k]_{3}\right) d \vartheta\left\{\begin{array}{l}
\left\{E_{m}\right. \\
\left\{F_{m}\right.
\end{array}\right\}\right\},
\end{aligned}
$$


where $p_{i}(\vartheta), i=1,2 \ldots 9$ and $q_{j}(\vartheta), j=1,2 \ldots 16$ are variable coefficients depending on the meridional coordinate $\vartheta$, which are specified in Appendix A. Submatrices $[k]_{i}$, whose elements are products of sine and cosine functions or their derivatives per $\vartheta$, are listed in Appendix C.

The system of three matrix equations (9) can be presented in a condensed form:

$$
\frac{\partial E_{S}}{\partial\{\delta\}}=[K]\{\delta\}
$$

where

$$
\{\delta\}^{T}=\langle\delta\rangle=\left\langle\left\langle A_{m}\right\rangle\left\langle B_{m}\right\rangle\left\langle C_{m}\right\rangle\left\langle D_{m}\right\rangle\left\langle E_{m}\right\rangle\left\langle F_{m}\right\rangle\right\rangle
$$

is the vector of Fourier coefficients, and:

$$
[K]=\left[\begin{array}{lll}
{[K]_{11}} & {[K]_{12}} & {[K]_{13}} \\
{[K]_{21}} & {[K]_{22}} & {[K]_{23}} \\
{[K]_{31}} & {[K]_{32}} & {[K]_{33}}
\end{array}\right]
$$

is the stiffness matrix. Submatrices $[K]_{i j}, i, j=1,2,3$, encompass the integrals in Eq. (9).

\subsection{GEOMETRIC STIFFNESS MATRIX}

Geometric stiffness matrix is derived from the strain energy component which is due to the pre-stressing, Eq. (3). Substituting expressions (7) for displacements into (3) and differentiating it per Fourier coefficients, the following three matrix equations are obtained:

$$
\begin{aligned}
& \left\{\begin{array}{l}
\frac{\partial E_{G}}{\left\{\partial A_{k}\right\}} \\
\left\{\partial B_{k}\right\}
\end{array}\right\}=\int_{-\vartheta_{0}}^{\vartheta_{0}}\left(c_{1}[k]_{1}+c_{2}[k]_{2}\right) d \vartheta\left\{\begin{array}{l}
\left\{A_{m}\right\} \\
\left\{B_{m}\right\}
\end{array}\right\}+\int_{-\vartheta_{0}}^{\vartheta_{0}} c_{8}[k]_{2} d \vartheta\left\{\begin{array}{l}
\left\{C_{m}\right\} \\
\left\{D_{m}\right\}
\end{array}\right\} \\
& +\int_{-\vartheta_{0}}^{\vartheta_{0}}\left(c_{9}\left([k]_{4}^{0}-[k]_{4}^{*}\right)+c_{10}[k]_{2}\right) d \vartheta\left\{\begin{array}{l}
\left\{E_{m}\right\} \\
\left\{F_{m}\right\}
\end{array}\right\} \\
& \left\{\begin{array}{l}
\frac{\partial E_{G}}{\left\{\partial C_{k}\right\}} \\
\left\{\partial D_{k}\right\}
\end{array}\right\}=\int_{-\vartheta_{0}}^{\vartheta_{0}} c_{8}[k]_{2} d \vartheta\left\{\begin{array}{l}
\left\{A_{m}\right\} \\
\left\{B_{m}\right\}
\end{array}\right\}+\int_{-\vartheta_{0}}^{\vartheta_{0}}\left(c_{3}[k]_{1}+c_{4}[k]_{2}+c_{5}\left([k]_{4}^{0}+[k]_{4}^{*}\right)\right) d \vartheta\left\{\begin{array}{l}
\left\{C_{m}\right\} \\
\left\{D_{m}\right\}
\end{array}\right\} \\
& +\int_{-\vartheta_{0}}^{\vartheta_{0}} c_{11}[k]_{2} d \vartheta\left\{\begin{array}{l}
\left\{E_{m}\right\} \\
\left\{F_{m}\right\}
\end{array}\right\} \\
& \left\{\begin{array}{l}
\frac{\partial E_{G}}{\left\{\partial E_{k}\right\}} \\
\left\{\partial F_{k}\right\}
\end{array}\right\}=\int_{-\vartheta_{0}}^{\vartheta_{0}}\left(c_{9}\left([k]_{4}^{*}-[k]_{4}^{0}\right)+c_{10}[k]_{2}\right) d \vartheta\left\{\begin{array}{l}
\left\{A_{m}\right\} \\
\left\{B_{m}\right\}
\end{array}\right\}+\int_{-\vartheta_{0}}^{\vartheta_{0}} c_{11}[k]_{2} d \vartheta\left\{\begin{array}{l}
\left\{C_{m}\right\} \\
\left\{D_{m}\right\}
\end{array}\right\} \\
& +\int_{-\vartheta_{0}}^{\vartheta_{0}}\left(c_{6}[k]_{1}+c_{7}[k]_{2}\right) d \vartheta\left\{\begin{array}{l}
\left\{E_{m}\right\} \\
\left\{F_{m}\right\}
\end{array}\right\}
\end{aligned}
$$


where variable coefficients $c_{i}(\vartheta), i=1,2 \ldots 11$ are specified in Appendix B, and submatrices $[k]_{i}$ are given in Appendix C.

The system of three matrix equations (13) can be presented in a condensed form following the layout of Eq. (10):

$$
\frac{\partial E_{G}}{\partial\{\delta\}}=[G]\{\delta\}
$$

where

$$
[G]=\left[\begin{array}{lll}
{[G]_{11}} & {[G]_{12}} & {[G]_{13}} \\
{[G]_{21}} & {[G]_{22}} & {[G]_{23}} \\
{[G]_{31}} & {[G]_{32}} & {[G]_{33}}
\end{array}\right]
$$

is the geometric stiffness matrix. Submatrices $[G]_{i j}, i, j=1,2,3$, now represent the integrals in Eqs. (13).

Tensional forces $N_{\vartheta}$ and $N_{\varphi}$ are absorbed in coefficients $c_{i}(\vartheta)$, Eqs. (B1), and both occur due to the internal pressure and the centrifugal load. Accordingly, the geometric stiffness matrix can be split into two matrices, i.e. one which is due to the internal pressure and the other related to the centrifugal forces, i.e.

$$
[G]=p[G]_{p}+\Omega^{2}[G]_{\Omega} .
$$

\subsection{MASS MATRICES}

Mass matrices are derived from the kinetic energy, Eq. (4). By substituting expressions (7) into (4), and differentiating the kinetic energy per Fourier coefficients, one obtains the following system of algebraic equations:

$$
\begin{aligned}
&\left\{\begin{array}{l}
\left.\frac{\partial E_{k}}{\left\{\partial A_{k}\right\}}\right\}= \\
\left\{\partial B_{k}\right\}
\end{array}\right\} \int_{-\vartheta_{0}}^{\vartheta_{0}} r\left(\omega^{2}+\Omega^{2} \sin ^{2} \vartheta\right)[k]_{2} d \vartheta\left\{\begin{array}{l}
\left\{A_{m}\right\} \\
\left\{B_{m}\right\}
\end{array}\right\} \\
&-2 \alpha \omega \Omega \int_{-\vartheta_{0}}^{\vartheta_{0}} r \sin \vartheta[k]_{2} d \vartheta\left\{\begin{array}{l}
\left\{C_{m}\right\} \\
\left\{D_{m}\right\}
\end{array}\right\} \\
&-\alpha \Omega^{2} \int_{0}^{2 \pi} r \sin \vartheta \cos \vartheta[k]_{2} d \vartheta\left\{\begin{array}{l}
\left\{E_{m}\right\} \\
\left\{F_{m}\right\}
\end{array}\right\}
\end{aligned}
$$




$$
\begin{aligned}
& \left\{\begin{array}{l}
\frac{\partial E_{k}}{\left\{\partial C_{k}\right\}} \\
\left\{\partial D_{k}\right\}
\end{array}\right\}=-2 \alpha \omega \Omega \int_{-\vartheta_{0}}^{\vartheta_{0}} r \sin \vartheta[k]_{2} d \vartheta\left\{\begin{array}{l}
\left\{A_{m}\right\} \\
\left\{B_{m}\right\}
\end{array}\right\} \\
& +\alpha\left(\omega^{2}+\Omega^{2}\right) \int_{-\vartheta_{0}}^{\vartheta_{0}} r[k]_{2} d \vartheta\left\{\begin{array}{l}
\left\{C_{m}\right\} \\
\left\{D_{m}\right\}
\end{array}\right\} \\
& +2 \alpha \omega \Omega \int_{-\vartheta_{0}}^{\vartheta_{0}} r \cos \vartheta[k]_{2} d \vartheta\left\{\begin{array}{l}
\left\{E_{m}\right\} \\
\left\{F_{m}\right\}
\end{array}\right\} \\
& \left\{\begin{array}{l}
\frac{\partial E_{k}}{\left\{\partial E_{k}\right\}} \\
\left\{\partial F_{k}\right\}
\end{array}\right\}=-\alpha \Omega^{2} \int_{-\vartheta_{0}}^{\vartheta_{0}} r \sin \vartheta \cos \vartheta[k]_{2} d \vartheta\left\{\begin{array}{l}
\left\{A_{m}\right\} \\
\left\{B_{m}\right\}
\end{array}\right\} \\
& +2 \alpha \omega \Omega \int_{-\vartheta_{0}}^{\vartheta_{0}} r \cos \vartheta[k]_{2} d \vartheta\left\{\begin{array}{l}
\left\{C_{m}\right\} \\
\left\{D_{m}\right\}
\end{array}\right\} \\
& +\alpha \int_{-\vartheta_{0}}^{\vartheta_{0}} r\left(\omega^{2}+\Omega^{2} \cos ^{2} \vartheta\right)[k]_{2} d \vartheta\left\{\begin{array}{l}
\left\{E_{m}\right\} \\
\left\{F_{m}\right\}
\end{array}\right\},
\end{aligned}
$$

where $\alpha=\pi \rho h a$. The three matrix Eqs. (17) can be represented in the form:

$$
\frac{\partial E_{k}}{\partial\{\delta\}}=\left(\Omega^{2}[B]+\omega \Omega[C]+\omega^{2}[M]\right)\{\delta\}
$$

where

$$
\begin{aligned}
{[B] } & =\left[\begin{array}{lll}
{[B]_{11}} & {[O]} & {[B]_{13}} \\
{[0]} & {[B]_{22}} & {[0]} \\
{[B]_{31}} & {[0]} & {[B]_{33}}
\end{array}\right] \\
{[C] } & =\left[\begin{array}{lll}
{[0]} & {[C]_{12}} & {[0]} \\
{[C]_{21}} & {[0]} & {[C]_{23}} \\
{[0]} & {[C]_{32}} & {[0]}
\end{array}\right] \\
{[M] } & =\left[\begin{array}{lll}
{[M]_{11}} & {[O]} & {[0]} \\
{[0]} & {[M]_{22}} & {[0]} \\
{[O]} & {[O]} & {[M]_{33}}
\end{array}\right]
\end{aligned}
$$

are mass matrices related to the centrifugal force $\left(\Omega^{2}\right)$, Coriolis force $(\omega \Omega)$, and the ordinary inertia force $\left(\omega^{2}\right)$. Submatrices $[B]_{i j},[C]_{i j}$ and $[M]_{i j}, i, j=1,2,3$ are specified in Appendix D. They all depend on the symmetric matrix $[k]_{2}$, Appendix C. Therefore, all mass matrices (19) are symmetric. 


\section{MATRIX EQUATION OF MOTION}

If a linear conservative dynamic system vibrates at its natural frequency, then it interchanges vibration energy from a purely potential state with the maximum strain energy, $E_{\text {smax }}$, to a purely kinetic state where the kinetic energy is maximum, $E_{k m a x}$. Hence, the difference of the maximum energies, $\Pi=E_{s \max }-E_{k \max }$, equals zero. If these energies are determined for approximated mode shapes, then the difference $\Pi$ is not zero. However, for a successful approximation of the true mode shape it should be as close to zero as possible.

In the considered case of a rotating toroidal shell the balance of energies reads:

$$
\Pi=E_{S}+E_{G}-E_{k} .
$$

All the terms on the right hand side are time-invariant due to the fact that fixed mode profiles rotate around the axis of symmetry of the torus [10]. Natural frequency is in fact the speed of this rotation. Then the integration over the circumferential coordinate eliminates temporal variations since it is irrelevant how the mode profile is positioned with reference to $\varphi=0$. Nevertheless, each particle on the shell still undergoes motions where minima and maxima of the displacement and velocity are interchanged. If the modes are determined approximately with truncated series, the governing equation of motion can still be obtained from the minimum total energy principle $[15,10]$ :

$$
\frac{\partial \Pi}{\partial\{\delta\}}=\frac{\partial E_{S}}{\partial\{\delta\}}+\frac{\partial E_{G}}{\partial\{\delta\}}-\frac{\partial E_{k}}{\partial\{\delta\}}=\{0\} .
$$

Taking into account relations (10), (14) with (16), and (18) respectively, one obtains the following matrix equation of natural vibrations:

$$
\left([K]+p[G]_{p}+\Omega^{2}\left([G]_{\Omega}-[B]\right)-\omega \Omega[C]-\omega^{2}[M]\right)\{\delta\}=\{0\} .
$$

The matrix $[C]$ multiplying the mixed $\omega \Omega$ term, which results from the Coriolis term in the kinetic energy expression (4), is the only one causing a bifurcation of natural frequencies. The geometric stiffness matrix $[G]_{\Omega}$ and the mass matrix $[B]$ are related to the centrifugal force with stiffening and softening effect respectively. The former is dominant with respect to the latter and, by increasing the rotational speed, the arithmetic mean of a bifurcated natural frequency is increased.

Since the considered toroidal shell is symmetric, Figure 1, the eigenvalue problem, Eq. (22), can be solved separately for the symmetric and antisymmetric natural modes. In the first case the vector of Fourier coefficients, Eq. (11), is reduced to:

$$
\{\delta\}_{s}^{T}=\left\langle\left\langle B_{m}\right\rangle\left\langle C_{m}\right\rangle\left\langle E_{m}\right\rangle\right\rangle
$$

while in the second case:

$$
\{\delta\}_{a}^{T}=\left\langle\left\langle A_{m}\right\rangle\left\langle D_{m}\right\rangle\left\langle F_{m}\right\rangle\right\rangle .
$$

Consequently, the corresponding rows and columns in the matrix equation (22) are excluded. 


\section{ILLUSTRATIVE EXAMPLE}

The vibration analysis of an open simply supported toroidal shell segment shown in Figure 1 is carried out next. The shell geometric and physical properties are the following: $R=1 \mathrm{~m}$, $a=0.4 \mathrm{~m}, h=0.01 \mathrm{~m}, E=2.1 \cdot 10^{11} \mathrm{~N} / \mathrm{m}^{2}, v=0.3$, and $\rho=7850 \mathrm{~kg} / \mathrm{m}^{3}$. The shell central angle in the meridional plane is $\vartheta_{0}= \pm 3 \pi / 4$.

The first six natural frequencies are listed in Table 1 and compared with those determined by the finite strip method (FSM) [12], and the finite element method (FEM) by employing two commercial software packages with different shell finite elements $[16,17]$. If the present rigorous Rayleigh-Ritz results are used as the referent ones, discrepancies of the FSM and FEM results are within $1 \%$.

Table 1 Natural frequencies of simply supported toroidal shell, $\omega[\mathrm{Hz}], R=1 \mathrm{~m}, a=0.4 \mathrm{~m}, h=0.01 \mathrm{~m}$,

$$
\vartheta_{0}=3 \pi / 4
$$

\begin{tabular}{ccccccc}
\hline Mode & $n$ & $\begin{array}{c}\text { Mode } \\
\text { type }\end{array}$ & RRM & $\begin{array}{c}\text { FSM (3q, 3f) } \\
150 \text { FS }\end{array}$ & $\begin{array}{c}\text { FEM } \\
\text { CATIA QD8 } \\
38 \times 176 \text { FE }\end{array}$ & $\begin{array}{c}\text { FEM } \\
\text { ABAQUS S4R } \\
38 \times 124 \text { FE }\end{array}$ \\
\hline 1 & 0 & Asym. & 47.64 & 47.98 & 47.62 & 47.70 \\
2 & 1 & Asym. & 173.84 & 173.94 & 173.80 & 173.91 \\
3 & 1 & Sym. & 383.35 & 383.30 & 383.19 & 384.81 \\
4 & 2 & Asym. & 416.75 & 416.63 & 416.44 & 417.71 \\
5 & 0 & Sym. & 429.79 & 429.67 & 429.49 & 431.24 \\
6 & 2 & Sym. & 447.58 & 447.38 & 447.09 & 450.68 \\
\hline
\end{tabular}

The isometric view and orthogonal projections of the first six natural modes generated by ABAQUS are shown in Figures 2 and 3, respectively. Displacement components of the shell cross-section determined by RRM are shown in Figure 4 . Displacements $U$ and $W$ are symmetric while $V$ is antisymmetric, with respect to the $y$-z plane, for modes 3,5 and 6 , resulting with symmetric modes. In case of modes 1,2 and 4, displacements $U$ and $W$ are antisymmetric and $V$ is symmetric, so that the modes are antisymmetric. This is indicated in Table 1. Natural modes determined by RRM and FEM are very similar.

In order to analyse the influence of the internal pressure on natural vibrations, the tensional forces are determined according to the procedure presented in Appendix E. Diagrams of tensional forces $N_{1}$ and $N_{2}$ due to pressure of 100 bar are shown in Figure 5 as functions of the meridional angle, $\vartheta$. Both forces are of the same order of magnitude. Deformation of the shell cross-section is shown in Figure 6. Variation of natural frequencies due to the variation of the internal pressure is shown in Figure 7. The FSM and FEM results are almost the same as the presented RRM results.

Tensional forces $N_{1}$ and $N_{2}$ due to the centrifugal load determined by the procedure presented in Appendix E for rotational speed of $50 \mathrm{rad} / \mathrm{s}$ are shown in Figure 8. The circumferential force, $N_{2}$, is much higher than the meridional force $N_{1}$. Their values determined by FSM and FEM are very close to the RRM results shown in Figure 8. Deformation of the shell cross-section is shown in Figure 9. 
Diagrams of natural frequencies for the circumferential wave number $n=0, \pm 1$ and \pm 2 , where the sign designates the forward and backward travelling modes, are shown in Figures 10, 11 and 12 respectively, as functions of the dimensionless rotation speed. The problem is also solved by FSM and FEM. In the former case, the values of natural frequencies are very close to those of RRM, whereas FEM results show some discrepancies.

The CPU time in the performed vibration analysis by three methods, Table 1, is about $7 \mathrm{~s}$ for RRM, $15 \mathrm{~s}$ for FSM, $70 \mathrm{~s}$ for FEM (CATIA) and $60 \mathrm{~s}$ for FEM (ABAQUS). Hence, the time saving by applying the proposed Rayleigh-Ritz method is considerable when compared to FEM analyses.

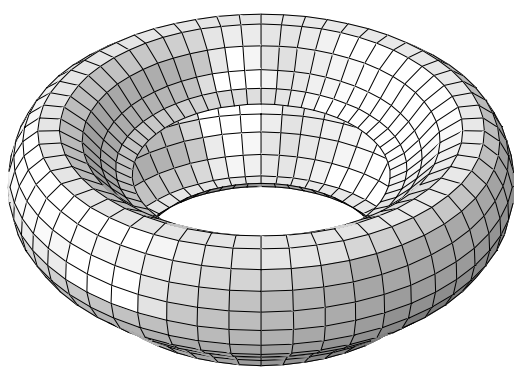

Mode 1, $n=0$

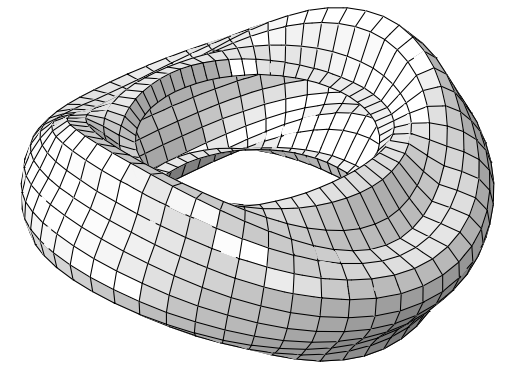

Mode 4, $n=2$

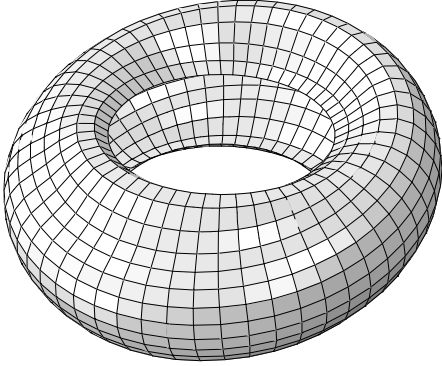

Mode 2, $n=1$

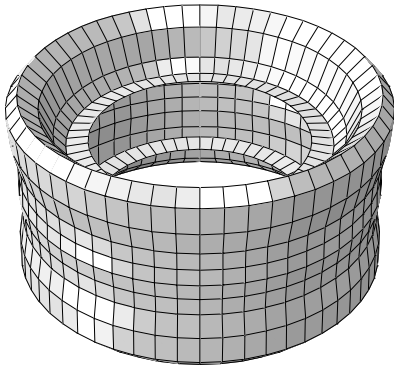

Mode 5, $n=0$

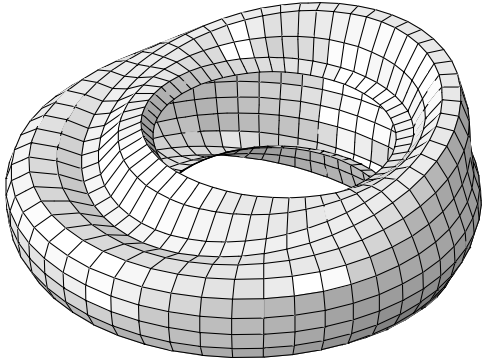

Mode 3, $n=1$

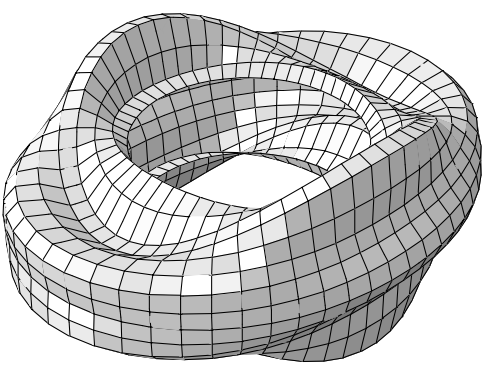

Mode 6, $n=2$

Fig. 2 Natural modes of simply supported toroidal shell (ABAQUS) 

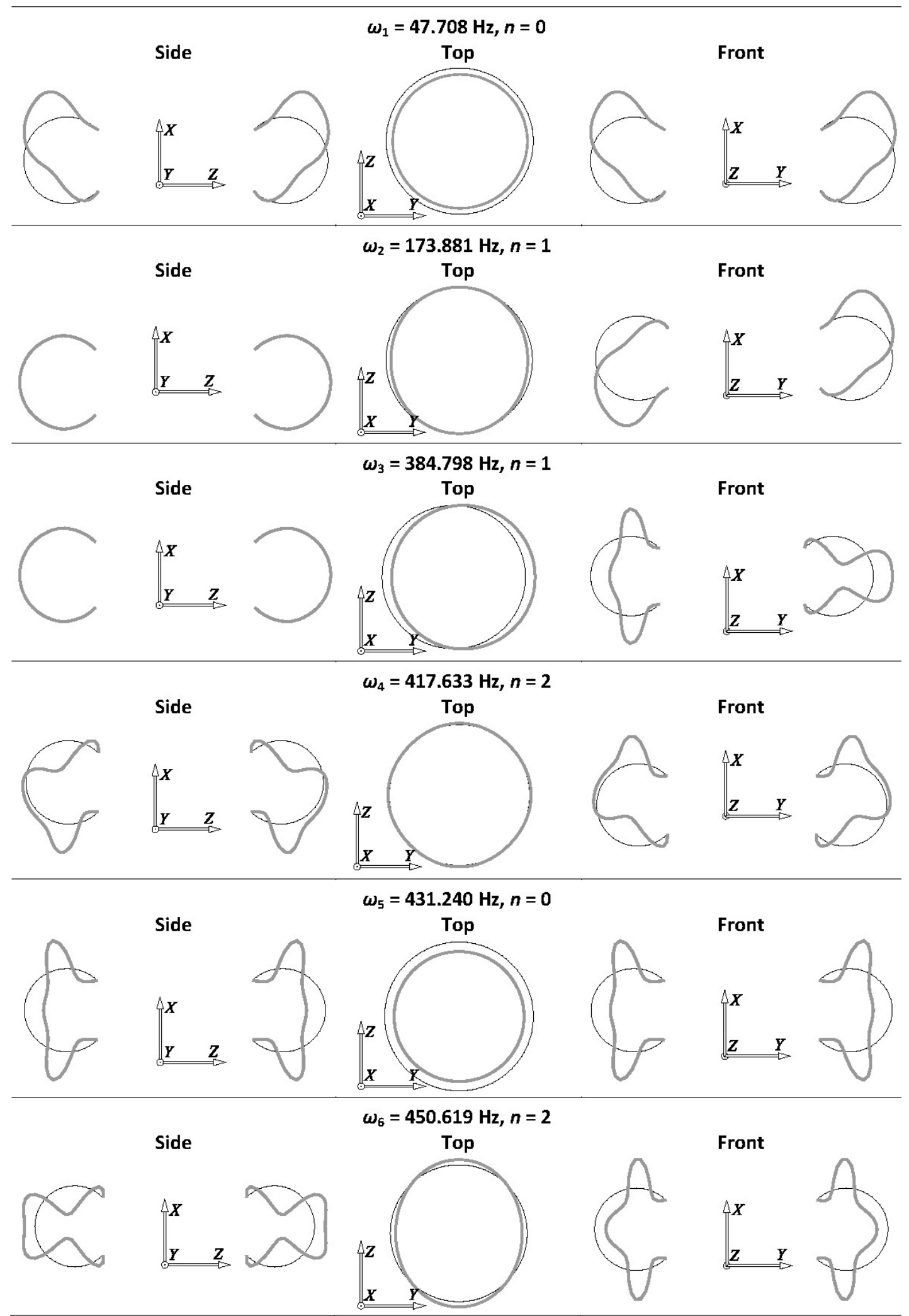

Fig. 3 Natural modes of simply supported toroidal shell in the orthogonal planes (ABAQUS) 


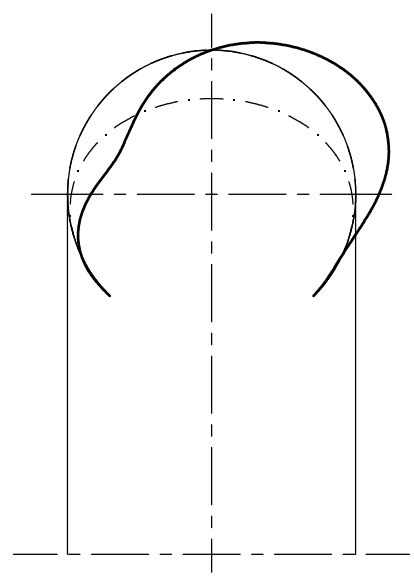

Mode $1, n=0$

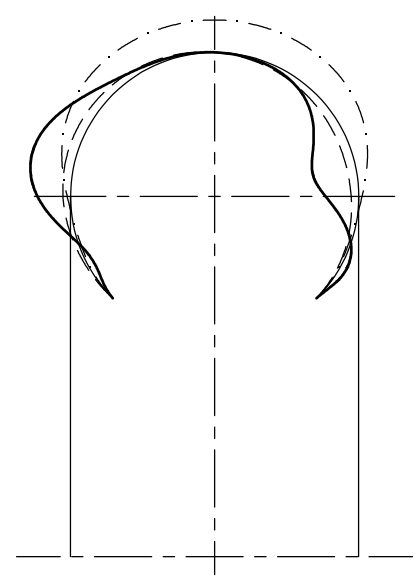

Mode 4, $n=2$

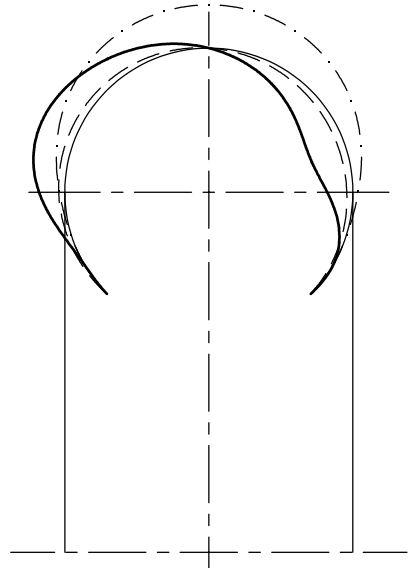

Mode 2, $n=1$

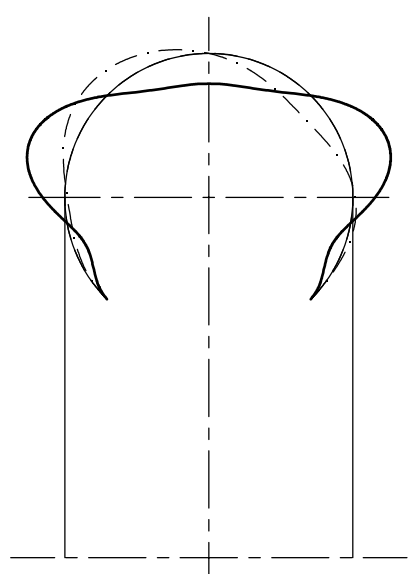

Mode 5, $n=0$

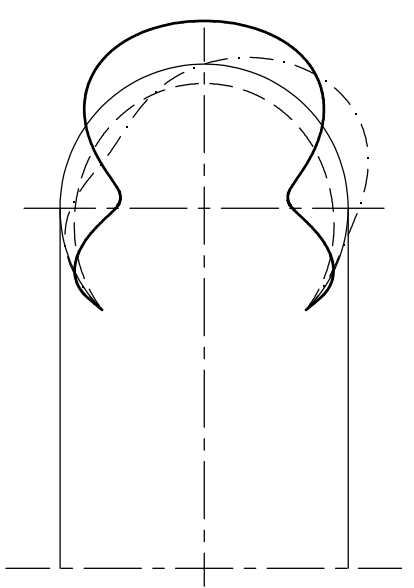

Mode $3, n=1$

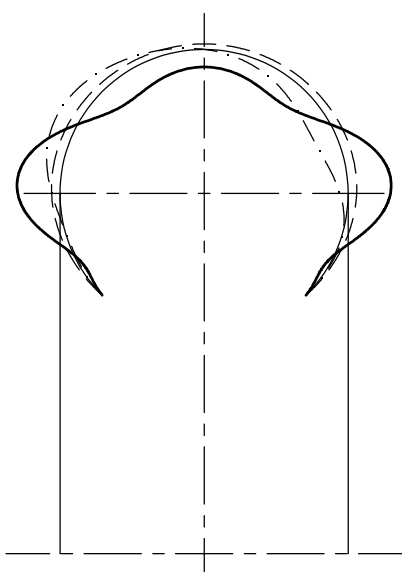

Mode $6, n=2$

Fig. 4 Cross-section displacements of simply supported toroidal shell: - - - U, - - V, --- W

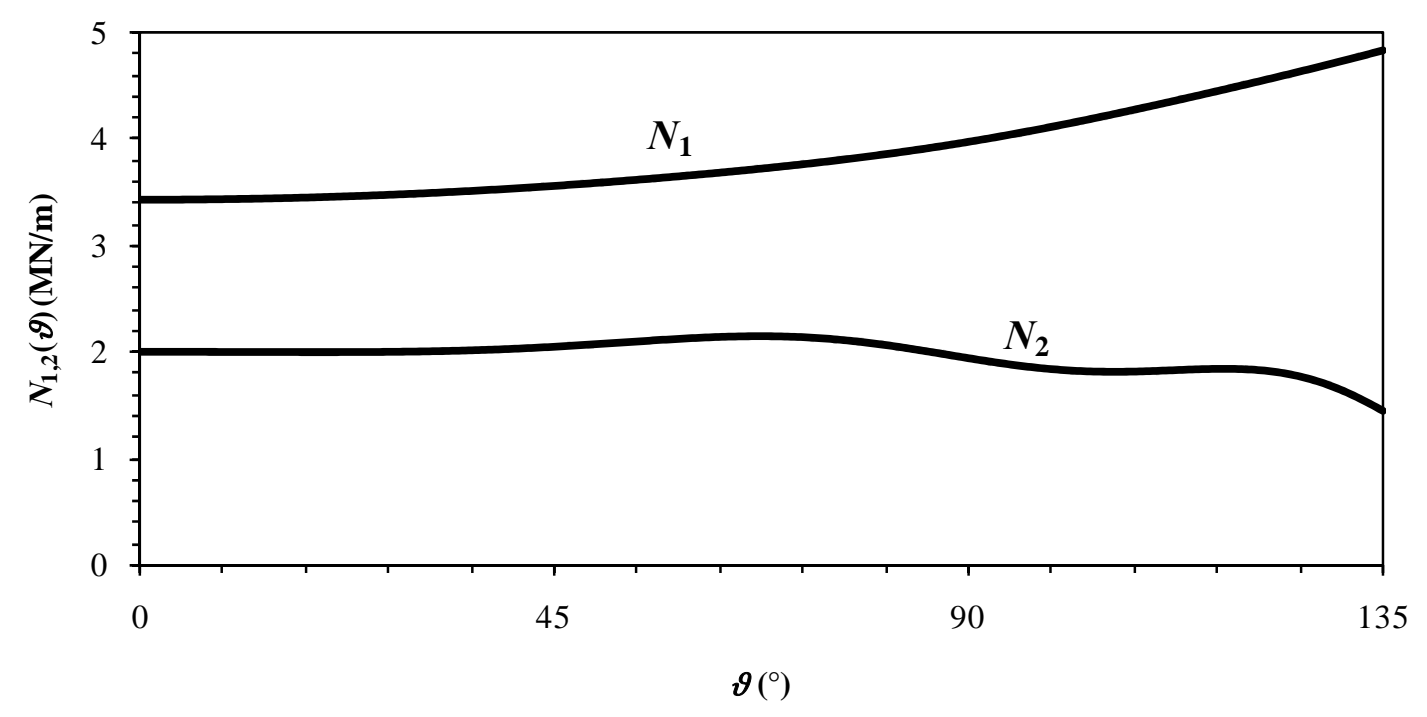

Fig. 5 Tensional forces due to pressure of 100 bar 


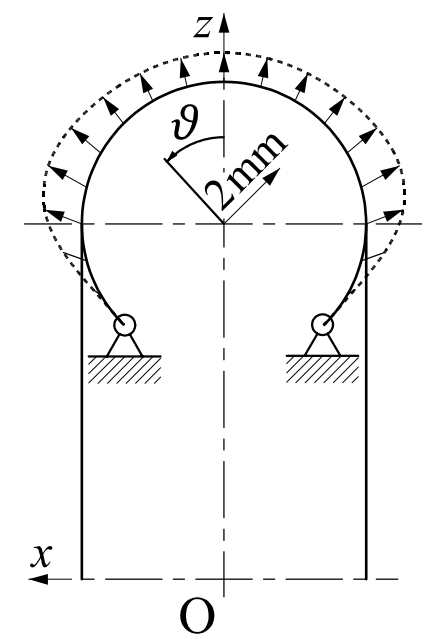

Fig. 6 Deformation of shell cross-section due to internal pressure of $100 \mathrm{bar}$

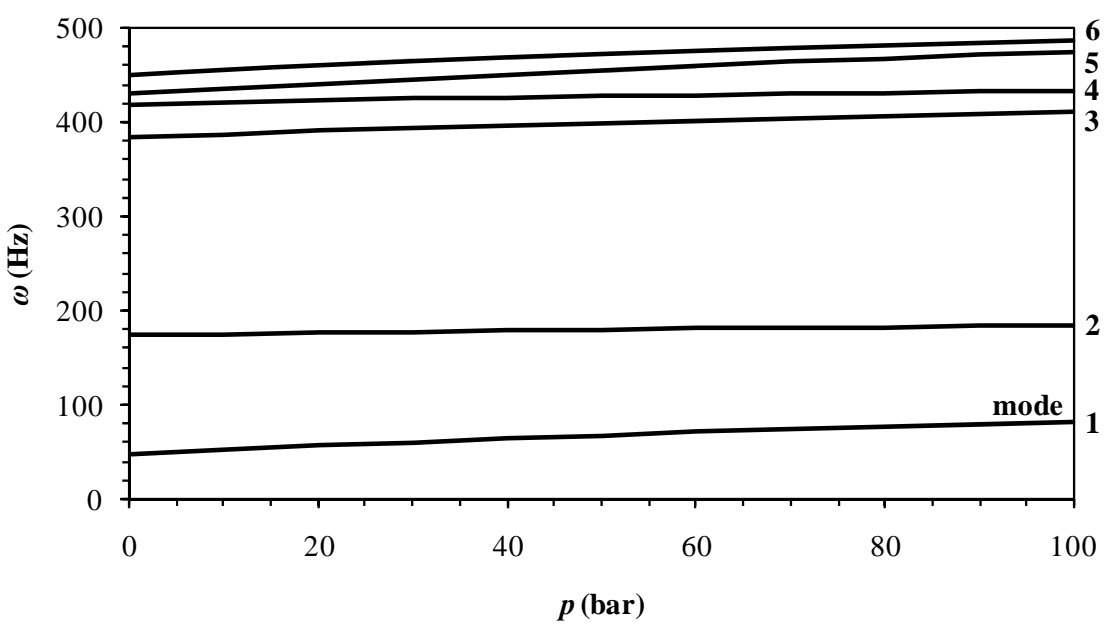

Fig. 7 Natural frequencies of the pressurized shell 

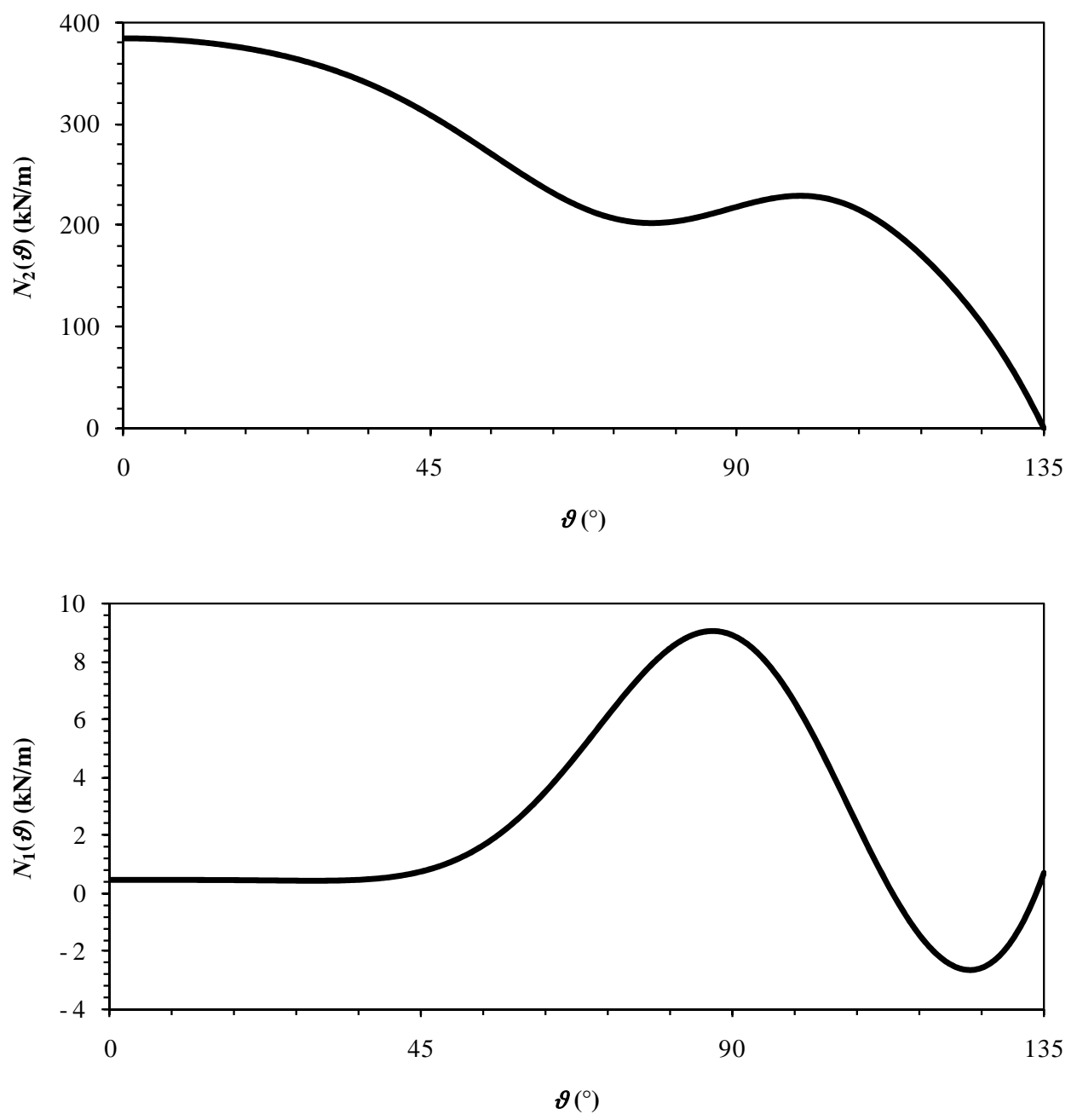

Fig. 8 Tensional forces of rotating toroidal shell, $\Omega=50 \mathrm{rad} / \mathrm{s}$

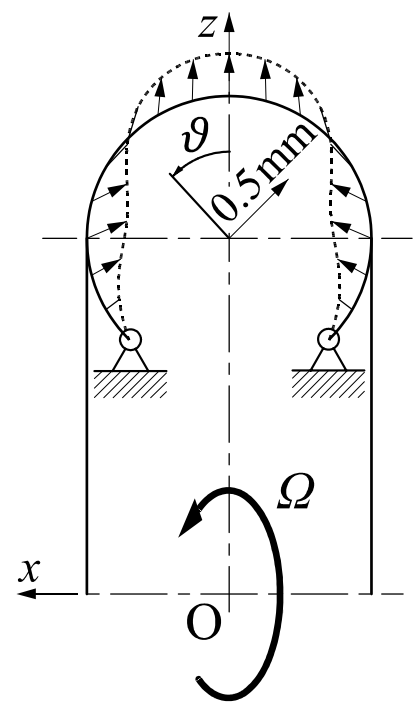

Fig. 9 Deformation of rotating shell cross-section, $\Omega=50 \mathrm{rad} / \mathrm{s}$ 
I. Senjanović, N. Alujević, I. Ćatipović, D. Čakmak, N. Vladimir, D-S Cho: Natural vibration analysis of pressurised and rotating toroidal shell segment by Rayleigh-Ritz method

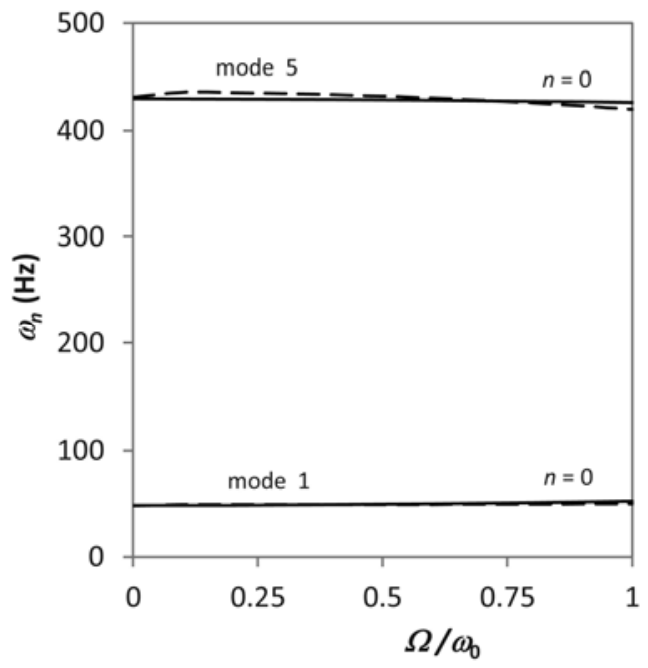

Fig. 10 Natural frequencies of rotating shell, $n=0,---R R M,--$ - FEM

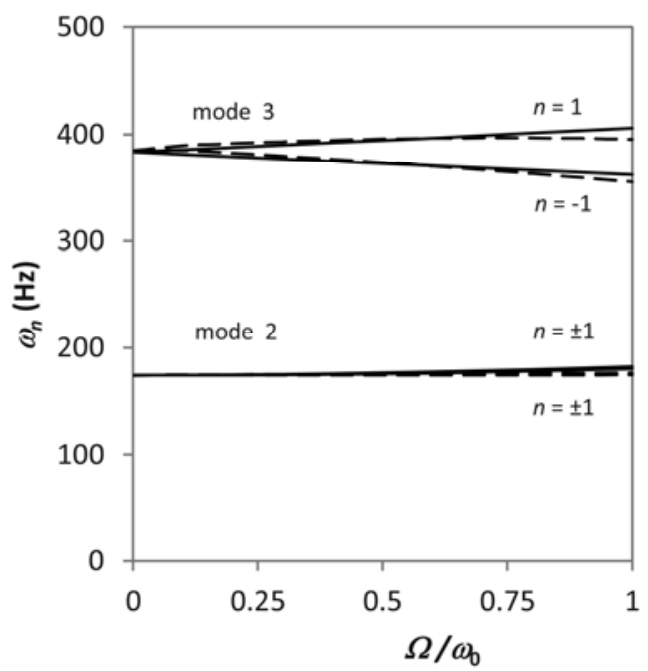

Fig. 11 Natural frequencies of rotating shell, $n= \pm 1,---R R M,--$ - FEM

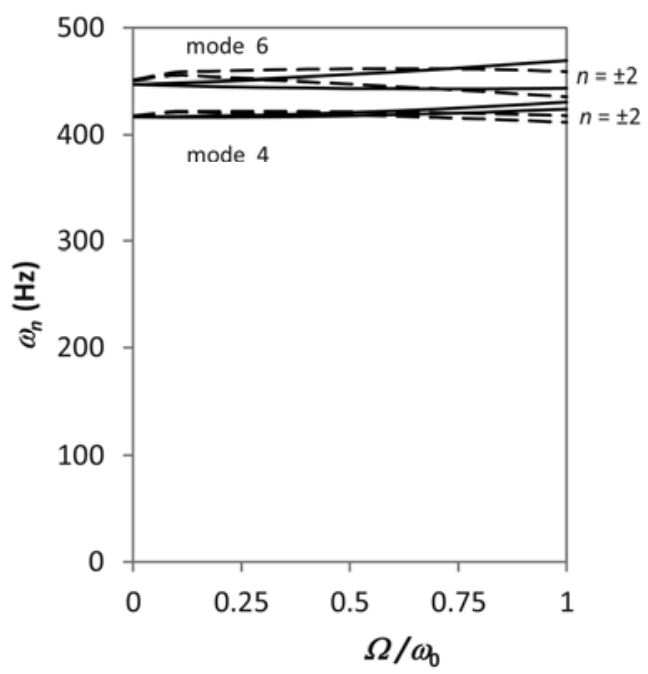

Fig. 12 Natural frequencies of rotating shell, $n= \pm 2,---R R M,--$ FEM 


\section{CONCLUSION}

A semi-analytical procedure for natural vibration analysis of simply supported toroidal shell segment by the Rayleigh-Ritz method and Fourier series is presented. The ordinary stiffness matrix, geometric stiffness matrix and three mass matrices, related to the pressure and centrifugal loads, Coriolis force and inertia load, all depend on a large number of variable coefficients and submatrices. In spite of this complexity, the procedure is presented in a consistent and physically transparent way, which is also easy for coding. The quadratic eigenvalue problem, $\operatorname{Det}\left[K\left(\omega^{0}, \omega^{1}, \omega^{2}\right)\right]_{d y n}=0$, can be solved with a commercial package as a polynomial eigenvalue problem. Forward and backward modes rotating in the circumferential direction and the corresponding natural frequencies are determined.

The convergence of the numerical results is very fast. Only 15 sine and cosine terms of the three sets of the Fourier series for displacement components is sufficient to achieve accurate results. As a result, savings of CPU time is considerable when compared to FEM analyses by models of couple of thousands d.o.f. in order to achieve the same accuracy.

Application of the Rayleigh-Ritz method for vibration analysis of toroidal shell segment with different boundary conditions is rather complicated. Therefore, such a problem can be solved via the finite strip method.

\section{ACKNOWLEDGEMENT}

This research has received funding from the European Union's Horizon 2020 research and innovation programme under the Marie Sklodowska-Curie grant agreement no. 657539. This investigation has also received funding within the international collaborative project Global Core Research Center for Ships and Offshore Plants (GCRC-SOP, No. 2011-0030669), established by the Republic of Korea Government (MSIP) through the National Research Foundation of South Korea (NRF).

\section{REFERENCES}

[1] V. Novozhilov, Thin Shell Theory, P. Noordhoff, Groningen, The Netherlands, 1964. https://doi.org/10.1007/978-94-017-5352-4

[2] I. Senjanović, Theory of Shells of Revolution, Ship Research Institute, Zagreb, 1972.

[3] W. Soedel, Vibrations of Shells and Plates (3 ${ }^{\text {rd }}$ edition), Marcel Dekker, Inc., New York, 2004.

[4] U. Eckstein, Nichtlineare Stabilitätsberechnung elastischer Schalentragwerke, TWM 83, Ruhr-Universität Bochum, Bochum, 1983.

[5] R. Harte \& U. Eckstein, Derivation of geometrically nonlinear finite shell elements via tensor notation, International Journal for Numerical Methods in Engineering, Vol. 23, pp. 367-384, 1986. https://doi.org/10.1002/nme.1620230304

[6] K.J. Bathe, Finite Element Procedures, Prentice-Hall, Englewood Cliffs, 1996. 
[7] N. Alujević, N. Campillo-Davo, P. Kindt, W. Desmet, B. Pluymers \& S. Vercammen, Analytical solution for free vibrations of rotating cylindrical shells having free boundary conditions, Engineering Structures, Vol. 132, pp. 152-171, 2017.

https://doi.org/10.1016/j.engstruct.2016.11.008

[8] N. Alujević, N. Campillo-Davo, P. Kindt, W. Desmet, B. Pluymers \& S. Vercammen, A simplified model of a rotating tire using cylindrical shells with free ends supported by an elastic foundation, In: Proceedings of ISMA2014, Katholieke Universiteit Leuven, Leuven, 2014.

[9] C. Gonzalez Diaz, P. Kindt, J. Middelberg, S. Vercammen, C. Thiry, R. Close \& J. Leyssens, Dynamic behaviour of a rolling tyre: experimental and numerical analyses, Journal of Sound and Vibration, Vol. 364, pp. 147-164, 2016.

https://doi.org/10.1016/j.jsv.2015.11.025

[10] I. Senjanović, N. Alujević, I. Ćatipović, D. Čakmak \& N. Vladimir, (2018a), Vibration analysis of rotating toroidal shell by the Rayleigh-Ritz method and Fourier series, Engineering Structures, Vol. 173, pp. 870-891, 2018.

https://doi.org/10.1016/i.engstruct.2018.07.029

[11] I. Senjanović, I. Ćatipović, N. Alujević, N. Vladimir \& D. Čakmak, (2018b), A finite strip for the vibration analysis of rotating cylindrical shells, Thin-Walled Structures, Vol. 122, pp. 158-172, 2018. https://doi.org/10.1016/j.tws.2017.10.017

[12] I. Senjanović, I. Ćatipović, N. Alujević, D. Čakmak \& N. Vladimir, A finite strip for the vibration analysis of rotating toroidal shell under internal pressure, ASME Journal of Vibration and Acoustics, 141, Paper No. 021013, 2019.

https://doi.org/10.1115/1.4041734

[13] S. Finnveden \& M. Fraggstedt, Waveguide finite elements for curved structures, Journal of Sound and Vibration, Vol. 312, pp. 644-671, 2008.

https://doi.org/10.1016/j.jsv.2007.11.020

[14] L. Gavrić, Finite element computation of dispersion properties of thin-walled waveguides, Journal of Sound and Vibration, Vol. 173, pp. 113-124, 1994.

https://doi.org/10.1006/jsvi.1994.1221

[15] R. Szilard, Theories and Applications of Plate Analysis, Classical, Numerical and Engineering Methods, John Wiley and Sons, Hoboken, New Jersey, USA, 2004. https://doi.org/10.1115/1.1849175

[16] Dassault Systèmes, CATIA V5R19 Documentation: Finite Element Reference Guide, 2007Vélizy-Villacoublay, France, 2007.

[17] Dassault Systèmes, ABAQUS 6.9 User's guide and theoretical manual, Hibbitt, Karlsson \& Sorensen, Inc., Providence, RI, 2009. 


\section{APPENDIX A: VARIABLE COEFFICIENTS OF THE STRAIN ENERGY}

$$
\begin{aligned}
& p_{1}=\pi\left(K+\frac{D}{a^{2}}\right) \frac{r}{a} \\
& p_{2}=\pi\left(K+\frac{D}{a^{2}}\right) \frac{a}{r}\left[\sin ^{2} \vartheta+\frac{1}{2}(1-v) n^{2}\right] \\
& p_{3}=-\pi\left(K+\frac{D}{a^{2}}\right) v \sin \vartheta \\
& p_{4}=\frac{1}{2} \pi(1-v)\left(K+\frac{D}{r^{2}} \cos ^{2} \vartheta\right) \frac{r}{a} \\
& p_{5}=\pi \frac{a}{r}\left\{K\left[n^{2}+\frac{1}{2}(1-v) \sin ^{2} \vartheta\right]+\frac{D}{r^{2}}\left[n^{2} \cos ^{2} \vartheta+\frac{1}{2}(1-v)\left(\frac{r}{a}\right)^{2} \sin ^{2} \vartheta\left(1-2 \frac{a}{r} \cos \vartheta\right)^{2}\right]\right\} \\
& p_{6}=-\frac{1}{2} \pi(1-v) \sin \vartheta\left[-K+\frac{D}{a r} \cos \vartheta\left(1-2 \frac{a}{r} \cos \vartheta\right)\right] \\
& p_{7}=\pi v n\left(K+\frac{D}{a r} \cos \vartheta\right) \\
& p_{8}=-\frac{1}{2} \pi(1-v) n\left(K+\frac{D}{a r} \cos \vartheta\right) \\
& p_{9}=-\frac{1}{2} \pi n \sin \vartheta\left\{K(3-v) \frac{a}{r}+\frac{D}{r^{2}}\left[2 \cos \vartheta-(1-v) \frac{r}{a}\left(1-2 \frac{a}{r} \cos \vartheta\right)\right]\right\} .
\end{aligned}
$$




$$
\begin{aligned}
& q_{1}=\pi \frac{D}{a^{2}} \frac{r}{a} \\
& q_{2}=\pi \frac{D}{a r}\left[\sin ^{2} \vartheta+2(1-v) n^{2}\right] \\
& q_{3}=\pi\left\{K\left(\frac{r}{a}+\frac{a}{r} \cos ^{2} \vartheta+2 v \cos \vartheta\right)+\frac{D}{r^{2}} \frac{a}{r} n^{2}\left[n^{2}+2(1-v) \sin ^{2} \vartheta\right]\right\} \\
& q_{4}=-\pi v \frac{D}{a^{2}} \sin \vartheta \\
& q_{5}=-\pi v \frac{D}{a r} n^{2} \\
& q_{6}=\pi(3-2 v) \frac{D}{r^{2}} n^{2} \sin \vartheta \\
& q_{7}=\pi \frac{D}{a^{2}} \frac{r}{a} \\
& q_{8}=\pi v \frac{D}{a^{2}} \sin \vartheta \\
& q_{9}=-\pi \frac{D}{a r}\left[\sin { }^{2} \vartheta+(1-v) n^{2}\right] \\
& q_{10}=\pi\left[K \frac{r}{a}\left(1+v \frac{a}{r} \cos \vartheta\right)+v \frac{D}{a r} n^{2}\right] \\
& q_{14}=-\pi \frac{D}{r^{2}} n\left[(1-v) \frac{r}{a}\left(1-2 \frac{a}{r} \cos \vartheta\right)-\cos \vartheta\right] \sin \vartheta \\
& q_{11}=-\pi\left[K\left(\frac{a}{r} \cos \vartheta+v\right)+(2-v) \frac{D}{r^{2}} n^{2}\right] \sin \vartheta \\
& q_{12}=-\pi v \frac{D}{a r} n \cos \vartheta \\
& q_{13}=\pi(1-v) \frac{D}{a r} n \cos \vartheta \\
& \left.q_{1-1}\left(\frac{a}{r} \cos \vartheta+v\right)+\frac{D}{r^{2}}\left[\frac{a}{r} n^{2} \cos \vartheta-(1-v) \sin ^{2} \vartheta\left(1-2 \frac{a}{r} \cos \vartheta\right)\right]\right\}
\end{aligned}
$$


10. APPENDIX B: VARIABLE COEFFICIENTS OF THE STRAIN ENERGY DUE TO PRE-STRESSING

$$
\begin{aligned}
& c_{1}=c_{3}=c_{6}=c_{9}=\pi \frac{r}{a} N_{\vartheta} \\
& c_{2}=\pi\left[\frac{r}{a} N_{\vartheta}+\frac{a}{r}\left(n^{2}+\sin ^{2} \vartheta\right) N_{\varphi}\right] \\
& c_{4}=\pi \frac{a}{r}\left[\sin ^{2} \vartheta N_{\vartheta}+\left(n^{2}+\cos ^{2} \vartheta\right) N_{\varphi}\right] \\
& c_{5}=\pi \sin \vartheta N_{\vartheta} \\
& c_{7}=\pi\left[\frac{r}{a} N_{\vartheta}+\frac{a}{r}\left(n^{2}+\cos ^{2} \vartheta\right) N_{\varphi}\right] \\
& c_{8}=-\pi \frac{a}{r} n \sin \vartheta N_{\varphi} \\
& c_{10}=-\pi \frac{a}{r} \sin \vartheta \cos \vartheta N_{\varphi} \\
& c_{11}=2 \pi \frac{a}{r} n \cos \vartheta N_{\varphi} .
\end{aligned}
$$

$N_{\vartheta}$ and $N_{\varphi}$ are tensional forces due to centrifugal load. 


\section{APPENDIX C: SUBMATRICES OF THE STIFFNESS MATRIX}

$$
\begin{aligned}
& {[k]_{1}=\left[\begin{array}{ll}
{\left[f_{k}^{\prime} f_{m}^{\prime}\right]} & {\left[f_{k}^{\prime} g_{m}^{\prime}\right]} \\
{\left[g_{k}^{\prime} f_{m}^{\prime}\right]} & {\left[g_{k}^{\prime} g_{m}^{\prime}\right]}
\end{array}\right]} \\
& {[k]_{2}=\left[\begin{array}{ll}
{\left[f_{k} f_{m}\right]} & {\left[f_{k} g_{m}\right]} \\
{\left[g_{k} f_{m}\right]} & {\left[g_{k} g_{m}\right]}
\end{array}\right]} \\
& {[k]_{4}^{0}=\left[\begin{array}{ll}
{\left[f_{k}^{\prime} f_{m}\right]} & {\left[f_{k}^{\prime} g_{m}\right]} \\
{\left[g_{k}^{\prime} f_{m}\right]} & {\left[g_{k}^{\prime} g_{m}\right]}
\end{array}\right]} \\
& {[k]_{4}^{*}=\left[\begin{array}{ll}
{\left[f_{k} f_{m}^{\prime}\right]} & {\left[f_{k} g_{m}^{\prime}\right]} \\
{\left[g_{k} f_{m}^{\prime}\right]} & {\left[g_{k} g_{m}^{\prime}\right]}
\end{array}\right]} \\
& {[k]_{5}^{0}=\left[\begin{array}{ll}
{\left[f_{k}^{\prime} f_{m}^{\prime \prime}\right.} & {\left[f_{k}^{\prime} g_{m}^{\prime \prime}\right.}
\end{array}\right]} \\
& {[k]_{5}^{*}=\left[\begin{array}{ll}
{\left[f_{k}^{\prime \prime} f_{m}^{\prime}\right]} & {\left[g_{k}^{\prime \prime} f_{m}^{\prime}\right.} \\
{\left[f_{k}^{\prime \prime} g_{m}^{\prime}\right]} & {\left[g_{k}^{\prime \prime} g_{m}^{\prime}\right]}
\end{array}\right]} \\
& {[k]_{6}^{0}=\left[\begin{array}{ll}
{\left[f_{k} f_{m}^{\prime \prime}\right]} & {\left[f_{k} g_{m}^{\prime \prime}\right]} \\
{\left[g_{k} f_{m}^{\prime \prime}\right]} & {\left[g_{k} g_{m}^{\prime \prime}\right]}
\end{array}\right]} \\
& {[k]_{6}^{*}=\left[\begin{array}{ll}
{\left[f_{k}^{\prime \prime} f_{m}\right]} & {\left[g_{k}^{\prime \prime} f_{m}\right]} \\
{\left[f_{k}^{\prime \prime} g_{m}\right]} & {\left[g_{k}^{\prime \prime} g_{m}\right]}
\end{array}\right]} \\
& {[k]_{7}=\left[\begin{array}{ll}
{\left[f_{k}^{\prime \prime} f_{m}^{\prime \prime}\right]} & {\left[f_{k}^{\prime \prime} g_{m}^{\prime \prime}\right]} \\
{\left[g_{k}^{\prime \prime} f_{m}^{\prime \prime}\right]} & {\left[g_{k}^{\prime \prime} g_{m}^{\prime \prime}\right]}
\end{array}\right]} \\
& {[k]_{3}=[k]_{4}^{0}+[k]_{4}^{*}} \\
& {[k]_{8}=[k]_{5}^{0}+[k]_{5}^{*}} \\
& {[k]_{9}=[k]_{6}^{0}+[k]_{6}^{*}}
\end{aligned}
$$




\section{APPENDIX D: SUBMATRICES OF THE MASS MATRICES}

$$
\begin{gathered}
{[B]_{11}=\alpha \int_{-\vartheta_{0}}^{\vartheta_{0}} r \sin ^{2} \vartheta[k]_{2} d \vartheta} \\
{[B]_{22}=\alpha \int_{-\vartheta_{0}}^{\vartheta_{0}} r[k]_{2} d \vartheta} \\
{[B]_{33}=\alpha \int_{-\vartheta_{0}}^{\vartheta_{0}} r \cos ^{2} \vartheta[k]_{2} d \vartheta} \\
{[B]_{13}=-\alpha \int_{-\vartheta_{0}}^{\vartheta_{0}} r \sin \vartheta \cos \vartheta[k]_{2} d \vartheta} \\
{[B]_{31}=[B]_{13}^{T}} \\
{[C]_{12}=-2 \alpha \int_{-\vartheta_{0}}^{\vartheta_{0}} r \sin \vartheta[k]_{2} d \vartheta} \\
{[C]_{23}=2 \alpha \int_{-\vartheta_{0}}^{\vartheta_{0}} r \cos \vartheta[k]_{2} d \vartheta} \\
{[C]_{21}=[C]_{12}^{T}, \quad[C]_{32}=[C]_{23}^{T}} \\
{[M]_{11}=[M]_{22}=[M]_{33}=\alpha \int_{-\vartheta_{0}}^{\vartheta_{0}} r[k]_{2} d \vartheta} \\
\alpha=\pi \rho h a
\end{gathered}
$$




\section{APPENDIX E: TENSIONAL FORCES DUE TO INTERNAL PRESSURE AND CENTRIFUGAL LOAD}

A rotating toroidal shell under internal pressure $p$ is exposed to the centrifugal load, Figure 1:

$$
q=\rho h \Omega^{2} r
$$

The meridional (tangential) and radial (normal) load components read:

$$
\begin{aligned}
& q_{1}=-\rho h \Omega^{2} r \sin \vartheta \\
& q_{n}=p+\rho h \Omega^{2} r \cos \vartheta .
\end{aligned}
$$

The shell deformation is symmetric with respect to the $x$-axis, i.e. $n=0$ and $u(\varphi, \vartheta)=U(\vartheta)$, $v(\varphi, \vartheta)=V(\vartheta)=0, \quad w(\varphi, \vartheta)=W(\vartheta)$. Function $U(\vartheta)$ is antisymmetric while $W(\vartheta)$ is symmetric with respect to the $y$-z plane, Figure 1. According to Eqs. (7) one can write:

$$
U(\vartheta)=\left\langle g_{m}\right\rangle\left\{B_{m}\right\}, W(\vartheta)=\left\langle f_{m}\right\rangle\left\{E_{m}\right\} .
$$

The total energy of the toroidal shell consists of the strain energy, Eq. (2), and the work of the loads $q_{1}$ and $q_{n}$, Eqs. (E2):

$$
\Pi=E_{S}^{0}+W_{q}
$$

where

$$
W_{q}=\int_{0}^{2 \pi} \int_{-\vartheta_{0}}^{\vartheta_{0}}\left(q_{1} U+q_{n} W\right) r a d \vartheta d \varphi=2 \pi a \int_{-\vartheta_{0}}^{\vartheta_{0}}\left(q_{1} U+q_{n} W\right) r d \vartheta .
$$

Using the principle of minimum total energy yields:

$$
\frac{\partial \Pi}{\partial\{\delta\}^{0}}=\frac{\partial E_{S}^{0}}{\partial\{\delta\}^{0}}+\frac{\partial W_{q}}{\partial\{\delta\}^{0}}=0,
$$

where

$$
\langle\delta\rangle^{0}=\left\langle\left\langle B_{m}\right\rangle\left\langle E_{m}\right\rangle\right\rangle
$$

is the vector of the unknown coefficients, Eqs. (E3).

According to Eq. (10), one can write for the first term in Eq. (E6):

$$
\frac{\partial E_{S}^{0}}{\partial\{\delta\}^{0}}=[K]^{0}\{\delta\}^{0}=2[K]\{\delta\}^{0}
$$

where $[K]$ is the reduced stiffness matrix, Eq. (12), due to reduced vector $\{\delta\}^{0}$. Relation $[K]^{0}=2[K]$ is a result of the circumferential energy integration. $[K]$ is determined generally for $n$-wave number, and in the case $n=0$, only one half of the strain energy is obtained [10]. The second term in (E6) is the load vector:

$$
\frac{\partial W_{q}}{\partial\{\delta\}^{0}}=\{F\}
$$


where

$$
\langle F\rangle=2 \pi a\left\langle\left\langle\rho h \Omega^{2} I_{m}^{(1)}\right\rangle\left\langle p I_{m}^{(2)}+\rho h \Omega^{2} I_{m}^{(3)}\right\rangle\right\rangle
$$

and

$$
\begin{aligned}
& I_{m}^{(1)}=-\int_{-\vartheta_{0}}^{\vartheta_{0}} r^{2} \sin \left(\eta_{m} \vartheta\right) \sin \vartheta d \vartheta \\
& I_{m}^{(2)}=\int_{-\vartheta_{0}}^{\vartheta_{0}} r \cos \left(\xi_{m} \vartheta\right) d \vartheta \\
& I_{m}^{(3)}=\int_{-\vartheta_{0}}^{\vartheta_{0}} r^{2} \cos \left(\xi_{m} \vartheta\right) \cos \vartheta d \vartheta .
\end{aligned}
$$

Hence, system of equations (E6) for the determination of the unknown coefficients reads:

$$
2[K]\{\delta\}^{0}=-\{F\} .
$$

Furthermore, the tensional strains can be determined [10]:

$$
\begin{aligned}
& \varepsilon_{\vartheta}=\frac{1}{a}\left(\frac{\partial U}{\partial \vartheta}+W\right) \\
& \varepsilon_{\varphi}=\frac{1}{r}(W \cos \vartheta-U \sin \vartheta)
\end{aligned}
$$

and finally the tensional forces by employing the Hooke's law are given by:

$$
\begin{aligned}
& N_{\vartheta}=\frac{E h}{1-v^{2}}\left[\frac{1}{a} \frac{d U}{d \vartheta}-\frac{v}{r} U \sin \vartheta+\left(\frac{1}{a}+\frac{v}{r} \cos \vartheta\right) W\right] \\
& N_{\varphi}=\frac{E h}{1-v^{2}}\left[\frac{v}{a} \frac{d U}{d \vartheta}-\frac{1}{r} U \sin \vartheta+\left(\frac{1}{r} \cos \vartheta+\frac{v}{a}\right) W\right] .
\end{aligned}
$$

